\title{
An effective DNA vaccine platform for Middle East respiratory syndrome coronavirus
}

\author{
Adam S. Cockrell ${ }^{1}$, Ralph S. Baric ${ }^{1,2}$ \\ ${ }^{1}$ Department of Epidemiology, ${ }^{2}$ Department of Microbiology and Immunology, University of North Carolina-Chapel Hill, Chapel Hill, North \\ Carolina, USA \\ Correspondence to: Ralph S. Baric. Department of Epidemiology, Department of Microbiology and Immunology, University of North Carolina-Chapel \\ Hill, Chapel Hill, North Carolina, USA. Email: rbaric@email.unc.edu. \\ Provenance: This is a Guest Editorial commissioned by Section Editor Binrong Zhou, MD, PhD (Department of Dermatology, The First Affiliated \\ Hospital of Nanjing Medical University, Nanjing, China). \\ Comment on: Muthumani K, Falzarano D, Reuschel EL, et al. A synthetic consensus anti-spike protein DNA vaccine induces protective immunity \\ against Middle East respiratory syndrome coronavirus in nonhuman primates. Sci Transl Med 2015;7:301ra132.
}

Submitted Oct 14, 2016. Accepted for publication Oct 18, 2016.

doi: 10.21037/atm.2016.11.40

View this article at: http://dx.doi.org/10.21037/atm.2016.11.40

Middle East respiratory syndrome coronavirus (MERS$\mathrm{CoV}$ ) is an ongoing emerging infectious disease across the Arabian Peninsula, with the majority of cases occurring in Saudi Arabia. Through September 23, 2016 the World Health Organization reported about 1,806 total cases, including 643 deaths from 27 countries (http://www.who. int/emergencies/mers-cov/en/). The disease is comprised of a lower respiratory infection wherein individuals exhibit pneumonia-like symptoms that often lead to multi-organ failure and death (1). In addition to close contact with infected camels, transmission from human-to-human most commonly occurs in the hospital setting through close contact between patients and hospital workers (1). MERS-CoV has also been isolated from objects within patient rooms including bed sheets, bed rails, and IV fluid hangers (2), which may all be potential sources of transmission. Several cases of MERS-CoV have been associated with travelers returning home from the Middle East and developing symptoms, including two cases of health care workers returning to the United States (3). The potential for global spread was recently illustrated by a South Korean national returning home from visiting the Arabian Peninsula in May, 2015, and initiating an outbreak that infected 186 people resulting in $20 \%$ mortality and a nationwide economic crisis (4). Nonetheless, MERS-CoV is not thought to be sustained in the human population through human-to-human transmission, but may instead be continuously re-introduced into the human population from a zoonotic source, most likely dromedary camels because of high seropositive rates in herds throughout the Middle East $(5,6)$. As camels are integral to the Saudi Arabian culture and economy, nationwide culling of camel herds is not feasible. Consequently, camel vaccination is being considered (7); however, therapeutic strategies have primarily focused on interfering with MERS-CoV infection in humans $(3,5)$.

To date, therapeutic strategies have been limited to a combination of ribavirin and interferon therapies with little evidence of efficacy (5). The predominant form of support continues to be palliative care including oxygen delivery through standard ventilation, or extracorporeal membrane oxygenation (ECMO) in more severe cases that have progressed to acute respiratory distress syndrome (ARDS). A number of human neutralizing antibodies directed to MERS-CoV are under development (5), and a phase I clinical trial has recently been initiated using a transchromosomal bovine production system to produce human polyclonal MERS-CoV antibodies (8). Considering that diagnosed cases of MERS-CoV typically present to hospital 5-10 days after initial infection, MERS-CoVdirected therapies, such as neutralizing antibodies, may be outside the therapeutic window for effectively quelling development of an immune pathologic ARDS disease phenotype. In hospital diagnosed cases, and early detected 
contact-traced infections, host-directed therapeutic interventions may be most beneficial. Nonetheless, neutralizing antibody therapy could provide a highly effective prophylactic treatment in hospital staff that work closely with infected individuals. While therapeutic intervention can be an effective strategy for immediate response to newly diagnosed cases in the hospital setting, widespread prophylactic treatment is not practical. The most effective prophylactic treatment would be development of an efficacious vaccine.

A MERS-CoV vaccine that can elicit a proper adaptive immune response to provide long-term protection may be most beneficial to the population across the Arabian Peninsula, especially when considering the persistent threat of re-introduction of MERS-CoV from dromedary camels. More than a decade of work on coronavirus vaccines have demonstrated that the spike protein, the major determinant of viral tropism, can elicit strong neutralizing antibody responses that are effective at protecting model organisms against challenge with homologous SARS-CoVs (9). Protective T cell responses targeting the nucleocapsid protein have also proven effective for preventing death, but not clinical disease in SARS-CoV infected rodents (10). Building upon knowledge from SARS$\mathrm{CoV}$ vaccines Muthumani et al. developed a novel DNA vaccine designed to elicit adaptive immune responses to the MERS-CoV spike protein (11). To enhance the success of their DNA vaccine Muthumani et al. built a consensus spike protein derived from MERS-CoV spike genomic sequences that represent both clades A and B. Vaccine efficacy was evaluated in three different mammalian species using a 3 dose delivery regimen (a prime and two boosts): mice ( 2 weeks intervals), three dromedary camels (4 weeks intervals), and rhesus macaques (3 weeks intervals) (11). An important gauge of vaccine efficacy is the cellular and humoral, adaptive immune responses that are elicited specifically to the MERS-CoV spike protein. Muthumani et al. identified a set of immunodominant MERS-CoVspecific spike epitopes that convincingly activate CD4+ and CD8+ T cells isolated from vaccinated mice and nonhuman primates (NHPs) (11). Cellular immune responses were not evaluated from vaccinated camels. Humoral immune responses were evaluated in mice, camels, and NHPs. The most effective measure of a humoral immune response to a viral antigen is the capacity of the vaccine to elicit antibodies that can neutralize viral infectivity on cellular assays. All DNA-spike vaccinated mice and NHPs generated antibodies capable of neutralizing the
EMC/2012 strain of MERS-CoV (11). The vaccine appears to be less effective in camels, which exhibited varying degrees of neutralizing activity, and a lack of neutralizing activity in 1 of 3 vaccinated camels. Muthumani et al. extended the humoral response observed for MERS-CoV to determine if serum could neutralize heterotypic spike proteins from human coronaviruses NL63 and HKU1, which are commonly associated with mild-upper respiratory tract infections (11). Serum from mice and NHPs could effectively neutralize both NL63 and HKU1 spike proteins in neutralization assays designed around pseudotyped lentiviral vector particles (11). However, as recognized by Muthumani et al., these assays may not reflect the activity that an antibody may exhibit against the spike protein presented in its native coronavirus context (11), and others have shown that cross neutralization between coronavirus genus and species is lacking when live virus is used (12). Overall, Muthumani et al. convincingly demonstrate that the consensus MERS-CoV DNA spike vaccine can elicit effective adaptive immune responses in both mice and NHPs, and to a lesser extent in camels.

The generation of adaptive immune across multiple mammalian species provides strong preclinical support for translation of the MERS-CoV DNA spike vaccine platform to human application. It also offers hope that a vaccine will soon be eminent for the prevention of MERS$\mathrm{CoV}$ infection both in humans and in reservoir animal species. However, the true barometer for vaccine efficacy is how well a vaccinated animal model is protected from development of disease symptoms following challenge of an infectious dose of MERS-CoV. This, in turn, can be complicated by the availability of animal models that accurately reflect MERS-CoV pathology in humans. Accordingly, proper model development requires an understanding of MERS-CoV pathology in humans. A clear picture of MERS-CoV pathogenesis in humans has been hampered by cultural constraints that prevent autopsy and procurement of human samples from fatal cases in Saudi Arabia. In January, 2016 a single publication described the histopathologic, immunohistochemical, and ultrastructural findings of a single fatal case of MERS$\mathrm{CoV}$ in the United Arab Emirates (13). MERS-CoV antigen was detected in the lungs having histopathology consistent with diffuse alveolar damage associated with ARDS, and no detection of MERS-CoV antigen in extrapulmonary tissues (13). Therefore, an efficacious vaccine platform could minimally be anticipated to protect a model organism from pathology consistent with MERS- 
CoV-induced ARDS. Recently, a new mouse model has been described which reproduces severe end stage lung disease phenotypes like ARDS, providing a new mouse model for prophylatic and therapeutic testing as well (14).

Muthumani et al. challenged vaccinated rhesus macaques with the MERS-CoV EMC/2012 strain (11). Animals vaccinated with the control vector exhibited clinical signs of disease, radiological evidence of lung disease, and histopathology indicative of viral pneumonia. The consensus DNA-spike vaccine protected NHPs from MERS-CoV-induced disease by reducing viral replication in the lungs of infected NHPs. Muthumani et al. demonstrate that a consensus MERS-CoV spike DNA vaccine platform can effectively elicit cellular and humoral immune responses that can protect NHPs from disease (11). The Muthumani et al. study provided preclinical efficacy data required by the Food and Drug Administration (FDA) to progress the vaccine forward into clinical trials. In February of 2016 the Walter Reed Army Institute of Research (WRAIR) announced initiation of the first phase I clinical trial to test the safety and immune response of this MERS-CoV DNA vaccine platform in people. The phase I clinical trial is being executed as collaboration between WRAIR and a DNA-based immunotherapy platform developed at Inovio. The Inovio platform comprises an algorithm optimized to determine an appropriate consensus sequence, as done for the MERS-CoV spike protein.

In 2016 Inovio utilized its proprietary platform to initiate phase I clinical DNA vaccine studies for MERS$\mathrm{CoV}, \mathrm{Zika}$ virus, and Ebola virus (http://www.inovio.com/). DNA vaccines provide a number of advantages over other vaccine approaches such as recombinant protein and liveattenuated vaccines: (I) development is more cost effective and easier; (II) there is no risk of reversion; (III) membrane protein targets (e.g., viral membrane glycoproteins) can be readily expressed in mammalian cells; and (IV) prior phase I clinical studies indicate that there is little inflammation/ toxicity associated with a DNA vaccine (15). Despite these advantages, there are no approved DNA vaccines for use in humans. DNA vaccines that elicit an effective immune response to infectious disease antigens continues to be a challenge in humans, as described in a number of former clinical trials (15). Tregoning et al. describe impediments in the DNA vaccine field that may have contributed to the lack of success in previous clinical trials (15), including: (I) the inability to adequately scale-up production; (II) differences in innate immune responses, between mammalian models and humans, which are required to initiate an appropriate adaptive immune response; (III) optimized methods of delivery (recognized as one of the biggest challenges in the field); and (IV) effective protein expression. The preclinical study by Muthumani et al. successfully addresses impediments 1,2 , and 4 by demonstrating the capacity to elicit effective adaptive immune responses in a small mammal (mice), a medium sized mammal (rhesus macaque), and a large mammal (dromedary camels) (11). To address impediment three Inovio developed a proprietary electroporation technology to achieve efficient delivery of vaccine plasmid DNA to the nucleus of cells, where expression of viral immunogen occurs. With numerous DNA vaccine trials underway, let us all hope that the efficacy of this vaccine platform in humans will be revealed within the next 2 years.

DNA vaccine platforms are an efficient means to rapidly respond to emerging infectious diseases (e.g., vaccines in clinical trials in 2016 for Zika virus, MERS-CoV, and Ebola virus). In the concluding remarks we would like to address concerns that may influence long-term efficacy and safety profiles of DNA vaccines. Early preclinical studies in animal models raised potential safety concerns (16) that were later adopted by the FDA as guidance for industry when addressing immunogenicity and safety of a DNA delivery platform (17). Some of the safety concerns raised include: (I) garnering an understanding of the biodistribution, persistence, and integration profiles potentially associated with the delivery of any DNA molecule into the nucleus of a mammalian cell; (II) potential for autoimmunity; and (III) transfer of antibiotic resistance genes to patients (17). Tregoning et al. indicate that due to the accumulated success of multiple phase I clinical studies over the last decade, the FDA guidelines have become more relaxed over time (15). Caveats in the FDA guidelines include waivers of biodistribution studies if the "novel gene is inserted into a plasmid vector previously documented to have an acceptable biodistribution/integration profile", and, that "integration studies are warranted only when plasmid persists in any tissue of any animal at levels exceeding 30,000 copies per $\mu \mathrm{g}$ of host DNA by study termination". Curiously, the preclinical research studies that established the precedent for assessing biodistribution/integration profiles were performed more than a decade ago, prior to initiation of most human clinical trials (16), and continue to be referenced as the established precedent for a lack of detectable biodistribution/integration (18). In looking toward the future and in light of the numerous DNA vaccine trials in progress, it might be prudent to re-evaluate 
this question using highly sensitive modern molecular biology technologies, commonly employed by the gene therapy field (19), to profile the human genome for putative sites of insertional mutagenesis.

\section{Acknowledgements}

Funding: This work was funded by grants AI110700, AI106772 and AI108197 from the National Institutes of Health.

\section{Footnote}

Conflicts of Interest: The authors have no conflicts of interest to declare.

\section{References}

1. van den Brand JM, Smits SL, Haagmans BL. Pathogenesis of Middle East respiratory syndrome coronavirus. J Pathol 2015;235:175-84.

2. Bin SY, Heo JY, Song MS, et al. Environmental Contamination and Viral Shedding in MERS Patients During MERS-CoV Outbreak in South Korea. Clin Infect Dis 2016;62:755-60.

3. Uyeki TM, Erlandson KJ, Korch G, et al. Development of Medical Countermeasures to Middle East Respiratory Syndrome Coronavirus. Emerg Infect Dis 2016;22.

4. Lee SI. Costly Lessons From the 2015 Middle East Respiratory Syndrome Coronavirus Outbreak in Korea. J Prev Med Public Health 2015;48:274-6.

5. de Wit E, van Doremalen N, et al. SARS and MERS: recent insights into emerging coronaviruses. Nat Rev Microbiol 2016;14:523-34.

6. Yusof MF, Eltahir YM, Serhan WS, et al. Prevalence of Middle East respiratory syndrome coronavirus (MERS$\mathrm{CoV}$ ) in dromedary camels in Abu Dhabi Emirate, United Arab Emirates. Virus Genes 2015;50:509-13.

7. Haagmans BL, van den Brand JM, Raj VS, et al. An orthopoxvirus-based vaccine reduces virus excretion after MERS-CoV infection in dromedary camels. Science 2016;351:77-81.

8. Luke T, Wu H, Zhao J, et al. Human polyclonal immunoglobulin $\mathrm{G}$ from transchromosomic bovines inhibits MERS-CoV in vivo. Sci Transl Med 2016;8:326ra21.

9. Graham RL, Donaldson EF, Baric RS. A decade after
SARS: strategies for controlling emerging coronaviruses. Nat Rev Microbiol 2013;11:836-48.

10. Zhao J, Zhao J, Mangalam AK, et al. Airway Memory CD4(+) T Cells Mediate Protective Immunity against Emerging Respiratory Coronaviruses. Immunity 2016;44:1379-91.

11. Muthumani K, Falzarano D, Reuschel EL, et al. A synthetic consensus anti-spike protein DNA vaccine induces protective immunity against Middle East respiratory syndrome coronavirus in nonhuman primates. Sci Transl Med 2015;7:301ra132.

12. Agnihothram S, Gopal R, Yount BL Jr, et al. Evaluation of serologic and antigenic relationships between middle eastern respiratory syndrome coronavirus and other coronaviruses to develop vaccine platforms for the rapid response to emerging coronaviruses. J Infect Dis 2014;209:995-1006.

13. Ng DL, Al Hosani F, Keating MK, et al. Clinicopathologic, Immunohistochemical, and Ultrastructural Findings of a Fatal Case of Middle East Respiratory Syndrome Coronavirus Infection in the United Arab Emirates, April 2014. Am J Pathol 2016;186:652-8.

14. Cockrell AS, Yount BL, Scobey T, et al. A mouse model for MERS coronavirus-induced acute respiratory distress syndrome. Nat Microbiol 2016;2:16226.

15. Tregoning JS, Kinnear E. Using Plasmids as DNA Vaccines for Infectious Diseases. Microbiol Spectr 2014;2.

16. Kutzler MA, Weiner DB. DNA vaccines: ready for prime time? Nat Rev Genet 2008;9:776-88.

17. USA FDA: Guidance for industry: considerations for plasmid DNA vaccines for infectious disease indications. Biotechnol Law Rep 2007;26:641-7.

18. Flingai S, Czerwonko M, Goodman J, et al. Synthetic DNA vaccines: improved vaccine potency by electroporation and co-delivered genetic adjuvants. Front Immunol 2013;4:354.

19. Paruzynski A, Arens A, Gabriel R, et al. Genome-wide high-throughput integrome analyses by nrLAM-PCR and next-generation sequencing. Nat Protoc 2010;5:1379-95.

Cite this article as: Cockrell AS, Baric RS. An effective DNA vaccine platform for Middle East respiratory syndrome coronavirus. Ann Transl Med 2016;4(24):499. doi: 10.21037/ atm.2016.11.40 